\title{
Há correlação entre o grau de resiliência e o impacto da fibromialgia na qualidade de vida?
}

\author{
Is there correlation between the degree of resilience and the impact of quality of life in \\ patients with fibromyalgia?
}

\begin{abstract}
RESUMO
Introdução: A fibromialgia (FM) é uma síndrome dolorosa caracterizada por dores musculares difusas e pontos musculares circunscritos dolorosos à digitopressão. A importância dessa síndrome é o impacto na qualidade de vida. Um dos possíveis influenciadores desse impacto pode ser a baixa resiliência dos pacientes. Objetivos: Medir a resiliência nos pacientes com FM; medir o grau de qualidade de vida de pacientes com FM; relacionar e comparar o grau de resiliência com variáveis demográficas, socioeconômicas e qualidade de vida. Material e métodos: A coleta de dados foi realizada no Ambulatório de Reumatologia do Conjunto Hospitalar de Sorocaba. Foram aplicados: questionário sociodemográfico, escala de resiliência de Wagnild e Young, questionário genérico de avaliação da qualidade de vida Medical Outcomes Study 36-item Short-form Health Survey. Para a análise, foi utilizado o coeficiente de correlação de Spearman. Resultados: Foram estudadas 26 mulheres, com idade média de 47,7 $\pm 11,6$ anos e escolaridade média de 8,2 anos de estudo. Houve predomínio de pacientes com resiliência baixa. As análises estatísticas não demonstraram nenhum parâmetro (idade, intensidade da dor, impacto da FM ou qualidade de vida) que se correlacionasse moderada ou fortemente com o grau de resiliência. Conclusão: Pacientes com FM atendidos em um serviço universitário têm baixo grau de resiliência, alto impacto da FM e qualidade de vida ruim.

Palavras-chave: fibromialgia; qualidade de vida; resiliência psicológica.
\end{abstract}

\begin{abstract}
Introduction: Fibromyalgia (FM) is a syndrome characterized by diffuse pain and tender points on digit pressure. The importance of this syndrome is its impact on quality of life. One possible influencers of this impact may be the low resilience of patients. Objectives: To measure the resilience in patients with FM; to measure the quality of life in patients with FM; and to relate and compare the degree of resilience with demographic and socioeconomic varieties and quality of life. Material and methods: Data collection was performed at the Rheumatology Outpatient Clinic of the Conjunto Hospitalar de Sorocaba. Demographic and social data questionnaire, Wagnild and Young resilience scale, and SF-36 quality of life questionnaire were applied. For the analysis it was used the Spearman correlation coefficient. Results: Twenty-six women were studied, mean age of $47.7 \pm 11.6$ years and average schooling of 8.2 years of study. There was a predominance of patients with low resilience. The statistical analysis did not observe any parameter (age, pain intensity, FM impact or quality of life) that correlates moderately or strongly with the degree of resilience. Conclusion: FM patients followed at a tertiary care setting have low degree of resilience, high impact of FM and poor quality of life.
\end{abstract}

Keywords: fibromyalgia; quality of life; resilience, psychological.

${ }^{1}$ Pontifícia Universidade Católica de São Paulo (PUC-SP), Faculdade de Ciências Médicas e da Saúde - Sorocaba (SP), Brasil. Contato: jemartinez@pucsp.br

Recebido em 17/11/2015. Aceito para publicação em 25/05/2016. 


\section{INTRODUÇÃO}

Conceitua-se fibromialgia (FM) como uma síndrome dolorosa, que acomete preferencialmente mulheres e que é caracterizada por dores musculares difusas e pontos dolorosos à digitopressão. ${ }^{1}$

A teoria fisiopatológica mais aceita é que se trata de uma síndrome de amplificação dolorosa com alteração no processamento da nocicepção no tocante ao sistema nervoso central. Associa-se também a uma resposta anormal aos estressores habituais do cotidiano evidenciada por alterações no eixo hipotálamo-hipofisário-adrenal. ${ }^{2}$ Portanto, pode-se considerar a FM como uma apresentação de resposta alterada ao estresse.

O quadro clínico inclui: dor musculoesquelética difusa, distúrbios do sono, fadiga, rigidez matinal de curta duração, sensação de edema e parestesias. A associação com outras síndromes de natureza funcional é frequente. Entre elas, é possível citar a depressão, a ansiedade, a cefaleia crônica e a síndrome do cólon irritável. Os sintomas alteram-se em intensidade de acordo com fatores moduladores como, por exemplo, alterações climáticas, grau de atividade física e estresse emocional. ${ }^{3-5}$

Quanto ao exame físico, a única alteração característica é a hipersensibilidade dolorosa à digitopressão em áreas musculares circunscritas e específicas. O número de regiões a ser pesquisado varia conforme alguns autores entre $12 \mathrm{e}$ 24 áreas. ${ }^{3-5} \mathrm{O}$ Comitê Multicêntrico para a Classificação da Fibromialgia do Colégio Americano de Reumatologia (ACR) padronizou a pesquisa de 18 áreas, denominadas de pontos dolorosos, por meio do termo em inglês tender points. ${ }^{6}$

A FM pode se apresentar isoladamente ou associada a outras patologias, como hipotireoidismo, lúpus eritematoso sistêmico e artrite reumatoide.

Um aspecto importante dessa síndrome é o impacto na qualidade de vida dos pacientes e familiares. Os principais determinantes desse impacto são os sintomas de dor e fadiga e a incapacidade funcional resultante. ${ }^{7}$

Em 1990, o ACR estabeleceu critérios de classificação com a intenção de uniformizar os parâmetros de inclusão em estudos científicos, mas que também têm sido usados para orientar diagnósticos em casos individuais. Eles incluem a presença de dor musculoesquelética difusa que envolve abaixo e acima da cintura, coluna vertebral e membros, por período superior a três meses, associados aos pontos dolorosos citados anteriormente.

Quando usados para diagnóstico, esses critérios provocam um grande número de falso-negativos e falso-positivos; existe até mesmo um questionamento sobre sua validade na literatura internacional. ${ }^{8}$ Acredita-se que a questão da contagem dos pontos dolorosos deva ser analisada com cautela. Alguns autores relacionam o número total desses pontos ao estresse, e não à gravidade da FM. ${ }^{8}$

Por causa das críticas aos critérios de classificação de 1990, foram apresentados novos critérios, agora de diagnósticos, pelo ACR. Esses critérios aguardam validação externa e aceitação por parte da comunidade médica. Houve a retirada da contagem de pontos dolorosos e a introdução de outros sintomas além da dor. Os critérios são compostos de dois índices: um índice de dor generalizada (WPI), no qual são assinaladas 19 possíveis regiões em que o paciente sente dor, com um escore que varia entre 0 e 19; e o índice da severidade dos sintomas (SS), que avalia a fadiga, os distúrbios do sono, os distúrbios cognitivos e os sintomas somáticos. O diagnóstico da FM é sugerido quando o WPI é maior que 7, associado ao SS maior que 5, ou WPI entre 3 e 6 , com SS maior que 9. ${ }^{9}$

Portanto, na prática clínica é fundamental que se valorize a presença dos outros sintomas e que se pondere individualmente o valor dos pontos dolorosos, tanto em relação ao número como em relação a sua distribuição. Entende-se, porém, que essa questão não está totalmente resolvida.

Resiliência é um termo oriundo da física e tem conceito psicológico. Definida como a capacidade do indivíduo de lidar com um contexto tipicamente desconfortável, de superação de condições adversas que significariam ameaça à sua qualidade de vida, ao seu desenvolvimento ou à sua saúde mental. Refere-se, portanto, à capacidade de adaptação a momentos adversos da vida.

Um indivíduo com alta resiliência, assim, tem a capacidade de lidar e de superar suas dores e problemas em geral com mais facilidade. Dessa maneira, a qualidade de vida é aumentada em igual proporção ao aumento da resiliência, posto que o sofrimento, o estresse e os outros males são diminuídos. A hipótese deste estudo é que há correlação inversa entre o grau de resiliência e o impacto da FM e correlação direta com a qualidade de vida das pacientes.

Sob esse aspecto, identificar pacientes com baixa resiliência é importante para que possamos trabalhar de modo que essa resiliência seja aumentada e o paciente tenha maior qualidade de vida.

Os objetivos deste trabalho foram: determinar o grau de resiliência dos pacientes com FM que são atendidos em um centro terciário de atendimento na cidade de Sorocaba (SP), e verificar se há correlação entre o grau da resiliência e o impacto da FM e da qualidade de vida dessas pacientes.

\section{MATERIAL E MÉTODOS}

Casuística: 30 pacientes que preenchem os critérios de classificação para FM do ACR e que são acompanhadas no Ambulatório de Reumatologia do Conjunto Hospitalar de Sorocaba/ Pontifícia Universidade Católica de São Paulo (CHS/PUC-SP).

\section{Instrumentos de avaliação}

1. Questionário sociodemográfico composto por variáveis como gênero, idade, raça, escolaridade e renda familiar;

2. Escala de resiliência Wagnild e Young (1993), constituída por 25 itens descritos de forma positiva, respondidos em uma escala tipo Likert variando de 1 (discordo totalmente) a 7 (concordo totalmente). As pontuações possíveis variam de 25 a 175 , com pontos mais altos refletindo maior resiliência. Depois de repetidas aplicações da escala de resiliência com uma variedade de amostras, 
os autores definiram que o índice geral de resiliência é composto pela soma dos escores de todos os itens. Índices superiores a 145 indicam resiliência elevada; entre 125 e 145, resiliência moderada; e as pontuações abaixo de 120 , resiliência baixa;

3. Escala analógica numérica de intensidade da dor: 0 é ausência de dor e 10 é dor insuportável;

4. Questionário de impacto da fibromialgia (FIQ), para avaliar o impacto da FM na qualidade de vida com questões relacionadas à capacidade funcional, ao impacto ocupacional e à intensidade dos sintomas. Seu escore total varia de 0 a 100, sendo 0 o menor impacto possível e 100 o maior;

5. Questionário genérico de avaliação da qualidade de vida Medical Outcomes Study 36-item Short-form Health Survey. Esse instrumento é composto de oito escalas: capacidade funcional, limitações físicas, estado geral de saúde, vitalidade, saúde mental, aspectos sociais, aspectos emocionais e dor. Cada escala apresenta um escore de $0-100$, em que 0 corresponde ao pior estado de saúde, e 100, ao melhor estado de saúde.

\section{Análises}

As variáveis idade, escore total do FIQ e escala do SF-36 foram correlacionadas por meio do coeficiente de correlação de Spearman.

\section{Aspectos éticos}

O protocolo e o termo de consentimento livre e esclarecido foram aprovados pelo Comitê de Ética e Pesquisa da Faculdade de Ciências Médicas e da Saúde da PUC-SP.

\section{RESULTADOS}

Foram estudadas 26 pacientes do gênero feminino,

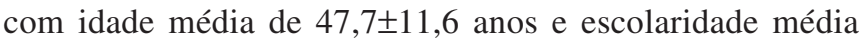
de 8,2 anos de estudo. A Tabela 1 apresenta a frequência de pacientes em cada categoria, segundo a intensidade da resiliência. Houve predomínio de pacientes com resiliência baixa, seguidas daquelas com grau moderado.

A Tabela 2 mostra o resultado do grau de resiliência e dos parâmetros de impacto da doença por intermédio do FIQ e da qualidade de vida pelas escalas do SF-36. A escala do SF-36 com pior qualidade de vida foi referente aos aspectos emocionais, e a melhor, acerca dos aspectos sociais, embora todas tenham apontado índices baixos de qualidade de vida.

As análises de correlação não observaram nenhum parâmetro (idade, intensidade da dor, impacto da FM ou qualidade de vida) que se correlacionasse moderada ou fortemente com o grau de resiliência.

Tabela 1. Avaliação do grau de resiliência em pacientes com fibromialgia.

\begin{tabular}{|lcc|}
\hline Grau de resiliência & Frequência & $\%$ \\
\hline Baixa & 13 & 50,0 \\
\hline Moderada & 11 & 42,3 \\
\hline Baixa & 2 & 7,7 \\
\hline
\end{tabular}

\section{DISCUSSÃO}

A natureza subjetiva dos sintomas da FM e a ausência de parâmetros mensuráveis para avaliar sua evolução fazem com que a medida do impacto da doença e o grau de prejuízo imposto à qualidade de vida passem a ser utilizados como parâmetros de desempenho. Assim, os fatores que podem influenciar a qualidade de vida passam a ser considerados alvo terapêutico, de maneira que sejam capazes de melhorar o objetivo final: a boa qualidade de vida. Neste estudo, optamos por utilizar o FIQ para medir esse impacto e o SF-36 para avaliar a qualidade de vida, pela alta aceitação que esses questionários têm na literatura cientifica e pela frequência com que são usados em estudos sobre FM.

A resiliência é um conceito muito interessante, já que emprestamos da física, particularmente do estudo dos materiais, uma ideia que pode ser aplicada no emocional humano. Levando em conta que a FM sofre grande influência dos aspectos emocionais, pela alta prevalência de doenças afetivas, sobretudo a ansiedade e a depressão, esse parâmetro poderia ser potencialmente útil na avaliação desses pacientes. ${ }^{9}{ }^{910}$ Hoje, sabe-se que o alto grau de catastrofização e a presença de ansiedade e depressão altas compõem um subgrupo de FM considerado mais severo e mais refratário ao tratamento convencional. ${ }^{11}$

As cinco características de resiliência, que servem como base conceitual para a escala de resiliência utilizada neste estudo, são:

1. perseverança, ou o ato de persistência: apesar da adversidade ou do desânimo, conota a vontade de continuar a luta para reconstruir a vida e seguir envolvido no meio da adversidade. Perseverança é a habilidade de continuar, embora haja contratempos (avaliada pelos itens 1, 2, 4, 14, 15, 20 e 23);

2. equanimidade: é a manutenção constante do temperamento e do humor independente da experiência que está sendo vivida. É avaliada pelos itens 7, 8, 11 e 12;

3. satisfação pessoal: refere-se a compreender o significado da vida e pode ser avaliada pelos itens 16, 21, 22 e 25;

Tabela 2. Dados referentes à resiliência média e ao impacto na qualidade de vida pelo questionário de impacto da fibromialgia e pelo questionário de qualidade de vida SF-36 de pacientes com fibromialgia.

\begin{tabular}{lc} 
Parâmetros & Média e desvio padrão \\
\hline Resiliência & $121,40(18,3)$ \\
\hline FIQ & $70,30(24,1)$ \\
\hline SF-36 & \\
\hline Estado geral da saúde & $22,90(18,2)$ \\
\hline Capacidade funcional & $23,07(18,7)$ \\
\hline Dor & $24,70(15,3)$ \\
\hline Vitalidade & $25,40(14,0)$ \\
\hline Aspectos sociais & $34,90(20,8)$ \\
\hline Aspectos emocionais & $16,60(30,1)$ \\
\hline Saúde mental & $32,90(23,4)$ \\
\hline FIQ: questionário de impacto da fibromialgia.
\end{tabular}

FIQ: questionário de impacto da fibromialgia. 
4. confiança em si mesmo: percepção de que a vida tem um propósito e o reconhecimento de que há algo para viver. Aqueles que são autoconfiantes acreditam em si mesmos. Eles reconhecem seus pontos fortes e capacidades pessoais e confiam neles, além de basearem-se em sucessos do passado para apoiar e talvez guiar suas ações. Essa percepção pode ser avaliada pelos itens $6,9,10,13,17,18$ e 24;

5. sentir-se bem só: percepção de que cada pessoa é única e que, apesar de algumas experiências poderem ser partilhadas, outras devem ser enfrentadas somente pelo indivíduo. É avaliada pelos itens 5, 3 e 19. Tal percepção dá o significado de liberdade e de que somos únicos e importantes. Com a solidão existencial, vem a sensação de exclusividade e, talvez, a de liberdade. ${ }^{12}$

Essas características podem ainda ser agrupadas em dois fatores: competência pessoal, que abrange autoconfiança, independência, decisão, invencibilidade, poder, inteligência e perseverança; e aceitação de si mesmo e da vida, refletindo a capacidade de adaptação, equilíbrio, flexibilidade e a perspectiva de vida estável, que corresponde à aceitação da vida e ao sentimento de paz, apesar das adversidades.

A casuística estudada determina algumas fragilidades para a generalização dos achados deste trabalho. Primeiramente, o $n$ pequeno indica o tamanho do ambulatório de nossa instituição. O alto grau de impacto e o baixo grau de qualidade de vida apontam o fato de ser um serviço terciário em que são atendidos os pacientes que se mostraram refratários aos tratamentos estabelecidos nos setores primário, secundário e mesmo terciário não universitários da saúde. Esses pacientes também apresentam grau de ansiedade e depressão ${ }^{10}$ bastante alto, o que os coloca no espectro mais severo dos sintomas da FM. ${ }^{11}$

Os parâmetros averiguados demonstram alto impacto da FM e baixa qualidade de vida. Seis escalas do SF-36 estão no quarto inferior da escala que vai de 0 a 100 , em que 0 é qualidade de vida muito ruim e 100 ótima qualidade de vida.

O impacto da FM tem sido bastante analisado na literatura, e, embora não cause nenhuma deformidade, a qualidade de vida desses pacientes equipara-se à de pacientes com artrite reumatoide. Esse achado foi constatado já em 1995 pelo nosso grupo de pesquisa e depois corroborado por estudos internacionais. ${ }^{7,13,14}$

Não se encontrou correlação significativa entre resiliência e os parâmetros de qualidade de vida. Por outro lado, nosso estudo alcançou índices de resiliência e de qualidade de vida bastante baixos, o que não permite a generalização. Assim, sugerimos que esta pesquisa seja reproduzida em cenários de atendimento de menor complexidade.

\section{CONCLUSÕES}

Conclui-se que pacientes com FM atendidos em um serviço universitário têm baixo grau de resiliência, alto impacto da FM e qualidade de vida ruim.

\section{REFERÊNCIAS}

1. Goldenberg DL. Fibromyalgia syndrome: an emerging but controversial condition. JAMA. 1987;257(20):2782-7.

2. Staud R. Fibromyalgia pain: do we know the source? Curr Opin Rheumatol. 2004;16(2):157-63.

3. Yunus MB, Masi AT, Calabro JJ, Miller KA, Feigenbaum SL. Primary fibromyalgia (fibrositis): clinical study of 50 patients with matched normal controls. Semin Arthritis Rheum. 1981;11(1):151-71.

4. Clark S, Campbell SM, Forehand ME, Tindall EA, Bennett RM. Clinical characteristics of fibrositis. A "blinded," controlled study using standard psychological tests. Arthritis Rheum. 1985;28(2):132-7.

5. Martinez JE, Atra E, Ferraz MB, Silva PSB. Fibromialgia: aspectos clínicos e socioeconômicos. Rev Bras Reumatol. 1992;32(5):225-30.

6. Wolfe F, Smythe HA, Yunus MB, Bennett RM, Bombardier C, Goldenberg DL, et al. The American College of Rheumatology 1990 Criteria for the Classification of Fibromyalgia. Report of the Multicenter Criteria Committee. Arthritis Rheum. 1990;33(2):160-72.

7. Martinez JE, Ferraz MB, Sato EI, Atra E. Fibromyalgia versus rheumatoid arthritis: a longitudinal comparison of the quality of life. J Rheumatol. 1995;22(2):270-4.

8. Wolfe F. The relation between tender points and fibromyalgia symptom variables: evidence that fibromyalgia is not a discrete disorder in the clinic. Ann Rheum Dis. 1997;56(4):268-71.

9. Wolfe F, Clauw DJ, Fitzcharles MA, Goldenberg DL, Katz RS, Mease P, et al. The American College of Rheumatology preliminary diagnostic criteria for fibromyalgia and measurement of symptom severity. Arthritis Care Res (Hoboken). 2010;62(5):600-10.

10. Yunus MB. Psychological aspects of fibromyalgia syndrome: a component of the dysfunctional spectrum syndrome. Baillieres Clin Rheumatol. 1994;8(4):811-37.

11. Martinez JE, Ferraz MB, Fontana AM, Atra E. Psychological aspects of Brazilian women with fibromyalgia. J Psychosom Res. 1995;39(2):167-74.

12. Giesecke T, Williams DA, Harris RE, Cupps TR, Tian X, Tian TX, et al. Subgrouping of fibromyalgia patients on the basis of pressure-pain thresholds and psychological factors. Arthritis Rheum. 2003;48(10):2916-22.

13. Wagnild GM, Young HM. Development and psychometric evaluation of the Resilience Scale. J Nurs Meas. 1993;1(2):165-78.

14. Burckhardt CS, Clark SR, Bennett RM. Fibromyalgia and quality of life: a comparative analysis. J Rheumatol. 1993;20(3):475-9. 